Research Paper

\title{
Management Of Synchronous Krukenberg Tumors From Gastric Cancer: a Single-center Experience
}

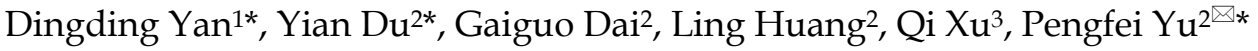 \\ 1. Department of Gynecologic oncology, Zhejiang Cancer Hospital, Hangzhou 310022, China; \\ 2. Department of Abdominal Surgery, Zhejiang Cancer Hospital, Hangzhou 310022, China; \\ 3. Department of Medical Oncology, Zhejiang Cancer Hospital, Hangzhou 310022, China. \\ *These authors contributed equally to this work. \\ $\square$ Corresponding author: Pengfei Yu, MD. Department of Abdominal Surgery, Zhejiang Cancer Hospital, 1\# Banshandong Road, Hangzhou 310022, China. Tel: \\ 86-571-88128041.Email:yupf@zjcc.org.cn \\ (c) Ivyspring International Publisher. This is an open access article distributed under the terms of the Creative Commons Attribution (CC BY-NC) license \\ (https://creativecommons.org/licenses/by-nc/4.0/). See http://ivyspring.com/terms for full terms and conditions.
}

Received: 2018.02.18; Accepted: 2018.07.16; Published: 2018.10.20

\begin{abstract}
Background: Whether metastasectomy improves prognosis of gastric cancer patients with ovarian metastases (Krukenberg tumors) is not clear. In this study, we examined the survival benefit of metastasectomy combined with chemotherapy for treatment of synchronous Krukenberg tumors from gastric cancer and identified the prognostic factors.

Methods: The subjects of this study were patients diagnosed as synchronous Krukenberg tumors of gastric origin in the period between December 2004 and December 2015. Patients were classified in accordance with treatment modality: metastasectomy group (metastasectomy combined with chemotherapy) and non-metastasectomy group (chemotherapy alone). Clinicopathological characteristics together with treatment records were investigated in detail and their relationship with survival outcomes was examined.

Results: Out of a total of 103 patients, 54 (52.4\%) underwent metastasectomy of Krukenberg tumors while 49 (47.6\%) patients had chemotherapy alone. Overall survival (OS) in the metastasectomy group was significantly longer than that in the non-metastasectomy group (18.9 months vs. 12.4 months, respectively; $P<0.001$ ). Metastasectomy (hazard ratio $[\mathrm{HR}] 0.486 ; 95 \%$ confidence interval $[\mathrm{Cl}]$ 0.323-0.729; $P<0.001)$, signet ring cells (HR 1.938; $95 \% \mathrm{Cl} 1.182-3.175 ; P=0.009)$, peritoneal carcinomatosis (HR 1.934; 95\% Cl 1.230-3.049; $P=0.004)$, expression of estrogen receptor- $\beta$ (ER- $\beta$ ) (HR $0.404 ; 95 \% \mathrm{Cl} 0.251-0.648 ; P<0.001)$, and progesterone receptor (PR) (HR 0.496; 95\% Cl 0.301-0.817; $P<0.001)$ were independent predictors of OS.

Conclusion: Metastasectomy combined with chemotherapy showed an association with survival benefit in patients with synchronous Krukenberg tumors from gastric cancer. Metastasectomy, expression of ER- $\beta$ and PR, peritoneal carcinomatosis, and signet ring cells were independent predictors of survival. Further prospective studies are warranted.
\end{abstract}

Key words: Krukenberg tumors; gastric neoplasm; metastasectomy; chemotherapy; prognosis

\section{Introduction}

Gastric cancer ranks as the fifth most common cancer and the third most common cause of cancer death worldwide [1]. Metastasis and high recurrence rate are the main reasons for poor prognosis of patients with gastric cancer. Krukenburg tumor is a metastatic tumor of the ovary that primarily arises from gastrointestinal tract [2]. It is reported that the incidence is approximately $0.3 \%$ to $6.7 \%$. However, incidence rates up to $33 \%-41 \%$ have been reported by autopsy studies $[3,4]$. Ovarian metastasis is correlated with worse prognosis; additionally, it is a main cause of treatment failure in patients with advanced gastric cancer [5]. Systemic chemotherapy provides symptom-relief and improves survival of patients 
with ovarian metastasis; however, treatment efficacy and overall survival are still unsatisfactory $[2,6]$. Some researchers have investigated the benefit of metastasectomy for synchronous Krukenberg tumors of gastric origin $[7,8]$. Nevertheless, there is no clear consensus on the benefit of ovarian metastasectomy, although it may be beneficial in a subset of patients [9]. A well-defined treatment strategy for synchronous Krukenberg tumors of gastric origin is yet to be established. In our series, we explored the association between metastasectomy of Krukenberg tumors, clinicopathological features, and overall survival in order to delineate a reasonable treatment strategy for these patients.

\section{Materials and Methods}

\section{Patients}

We retrospectively reviewed the clinical data pertaining to gastric cancer patients with synchronous ovarian metastasis who underwent metastasectomy or received systemic chemotherapy as the initial treatment at the Zhejiang Cancer Hospital (Hangzhou, China) between December 2004 and December 2015.

The inclusion criteria were as follows: histologically confirmed gastric adenocarcinoma; age range of patients: $18-75$ years; Eastern Cooperative Oncology Group (ECOG) performance status (PS) 0-1; technically resectable disease (synchronous Krukenberg tumor without peritoneal metastasis or with local peritoneal dissemination); adequate organ function (alanine transaminase and aspartate transaminase level < twice the normal upper limit (NUL); serum total bilirubin $<1.5$ times the NUL; serum creatinine $<1.25$ times the NUL; platelet count $\geq 100 \times 10^{9} / \mathrm{L}$; absolute granulocyte counts $\geq 1.5 \times 10^{9} / \mathrm{L}$; and hemoglobin level $\geq 90 \mathrm{~g} / \mathrm{L}$ ). We excluded gastric cancer patients with extensive peritoneal dissemination and other distant metastasis. The study was approved by the institutional ethics review board of the Zhejiang Cancer Hospital.

\section{Treatment and Assessment}

Based on the treatment modality, patients were categorized into two groups: metastasectomy group (patients who received chemotherapy combined with metastasectomy) and non-metastasectomy group (patients who received chemotherapy only).

All patients underwent imaging examinations to evaluate the extent of disease and resectability. The decision to perform metastasectomy was based on consensual opinion after comprehensive assessment of patient by a multidisciplinary team. Postoperative residual disease state in individual patients was categorized as gross residual disease (R2), positive margin of resection (R1), or complete resection with negative margins (R0). Postoperative complications were defined prospectively as any deviation from a pre-determined post-operative course within 30 days of surgery and classified according to the Clavien-Dindo severity classification (CDSC)[10]. Adverse events were evaluated using the Common Terminology Criteria for Adverse Events, version 3.0 (CTCAE, Ver. 3.0).

\section{Follow-up}

Patients underwent routine follow-up evaluation once every 3 months for the first 3 years, followed by once every 6 months during 3 to 5 years. Overall survival (OS) was defined as the time interval from the date of pathological diagnosis of gastric cancer to the date of death or most recent follow-up. The cutoff date for OS was December 31, 2017.

\section{Statistical Analysis}

Between-group differences with respect to continuous or discrete variables were assessed by two-tailed Student $t$-test or Chi-squared test, respectively. Survival analysis was performed using Kaplan-Meier method, and between-group differences with respect to OS were assessed using log-rank test. Cox proportional hazards model was used for multivariate analysis to identify independent prognostic factors. SPSS 19.0 for Windows (IBM Corporation, Armonk, NY, USA) was used for statistical analyses. $\mathrm{P}$ values $<0.05$ were considered indicative of statistically significant between-group difference.

\section{Results}

\section{Patient characteristics}

From December 2004 to December 2015, a total of 4923 female patients with gastric cancer were treated at the Zhejiang Cancer Hospital in Hangzhou, China. Of these, 272 patients had synchronous ovarian metastasis. 169 patients were excluded because they had extensive peritoneal dissemination and/or other distant metastasis.

The median duration of follow-up in the entire study cohort was 37.1 months (range, 2-71 months). Median age at study participation was 41.5 years (range, 18-65 years) and mean size of Krukenberg tumors was $7.9 \mathrm{~cm}$ (range, 2-21 cm). Fifty-four patients had received chemotherapy plus metastasectomy (metastasectomy group), while 49 patients had received chemotherapy alone (non-metastasectomy group).

Clinicopathological characteristics and features of synchronous ovarian metastasis from gastric cancer were enumerated in Table 1. The two groups were 
comparable with respect to tumor location, differentiation, ascites, tumor marker levels, TNM stage and expressions of ER and PR.

\section{Treatment outcome and prognostic factors}

The median OS in the entire cohort was 15.8 months (95\% confidence interval [CI], 14.2-17.4 months). The median OS in the metastasectomy group (18.9 months [95\% CI, 16.5-21.3 months]) was significantly better than that in the non-metastasectomy group (12.4 months [95\% CI, 10.8-14.1 months]; $P<0.001$ [Figure 1]).

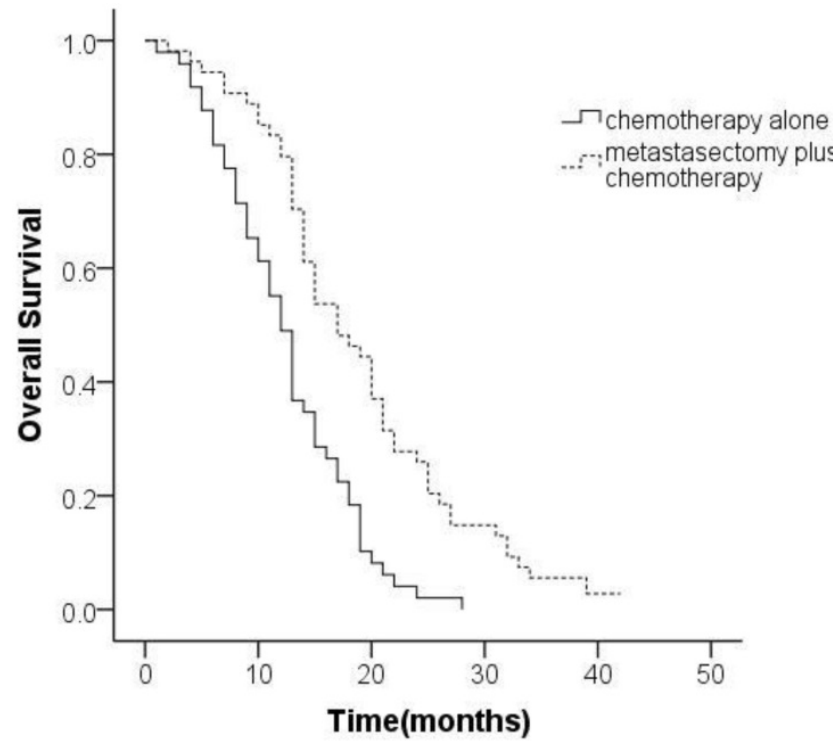

Figure 1. Kaplan-Meier analysis of overall survival based on treatment for gastric cancer patients with synchronous Krukenberg tumors.

Univariate analysis revealed a significant association between metastasectomy, peritoneal carcinomatosis, ascites, signet ring cells, expressions of ER- $\beta$ or PR, serum levels of CA19-9 and survival. After adjustment for covariates on multivariate analysis, metastasectomy (hazard ratio [HR] 0.335; 95\% CI $0.215-0.522 ; \quad P<0.001)$, peritoneal carcinomatosis (HR 2.212; 95\% CI 1.387-3.534; $P=0.001)$, signet ring cell (HR 1.938; 95\% CI 1.182-3.175; $P=0.009$ ), ER- $\beta$ positivity (HR 0.510; $95 \%$ CI 0.318-0.816; $P=0.005$ ), and PR positivity (HR 0.554; 95\% CI $0.340-0.901 ; P=0.017$ ) were found as independent predictors of overall survival (Table 2).

Table 1. Clinical characteristics of 103 patients with synchronous Krukenberg tumor

\begin{tabular}{|c|c|c|c|}
\hline Variable & $\begin{array}{l}\text { Metastasectomy plus } \\
\text { chemotherapy }(\mathrm{n}=54)\end{array}$ & $\begin{array}{l}\text { Chemotherapy } \\
\text { alone }(n=49)\end{array}$ & $P$-value \\
\hline Median age & $42.7(24-65)$ & $40.3(18-62)$ & 0.373 \\
\hline Tumor size $(\mathrm{cm})$ & $8.7(2.0-21.0)$ & $7.2(2.2-18.0)$ & 0.221 \\
\hline Laterality & & & 0.906 \\
\hline Bilateral & 38 & 35 & \\
\hline Unilateral & 16 & 14 & \\
\hline Peritoneum metastasis & & & 0.111 \\
\hline No & 38 & 41 & \\
\hline Yes & 16 & 8 & \\
\hline Signet-ring cells & & & 0.109 \\
\hline Positive & 40 & 29 & \\
\hline Negative & 14 & 20 & \\
\hline Differentiation & & & 0.232 \\
\hline Well and moderately & 10 & 5 & \\
\hline Poorly & 44 & 44 & \\
\hline Ascites & & & 0.362 \\
\hline No & 20 & 14 & \\
\hline Yes & 34 & 35 & \\
\hline ER & & & 0.313 \\
\hline Positive & 24 & 17 & \\
\hline Negative & 30 & 32 & \\
\hline PR & & & 0.282 \\
\hline Positive & 16 & 10 & \\
\hline Negative & 38 & 39 & \\
\hline Serum CEA (ng/mL) & & & 0.797 \\
\hline Normal & 43 & 40 & \\
\hline$>5$ & 11 & 9 & \\
\hline Serum CA19-9 (U/mL) & & & 0.438 \\
\hline Normal & 37 & 30 & \\
\hline$>39$ & 17 & 19 & \\
\hline Serum CA125 (U/mL) & & & 0.220 \\
\hline Normal & 24 & 16 & \\
\hline$>35$ & 30 & 33 & \\
\hline
\end{tabular}

Table 2. Univariate and multivariate analysis of prognostic factors for OS

\begin{tabular}{|c|c|c|c|c|}
\hline \multirow[b]{2}{*}{ Variable } & \multicolumn{2}{|l|}{ Univariate } & \multicolumn{2}{|l|}{ Multivariate } \\
\hline & HR $(95 \%$ CI) & $P$-value & $\operatorname{HR}(95 \% \mathrm{CI})$ & $P$-value \\
\hline Age ( $\geq 50$ years $)$ & $0.855(0.527-1.385)$ & 0.524 & - & - \\
\hline Size of tumor $(<5 \mathrm{~cm})$ & $0.958(0.637-1.439)$ & 0.835 & - & - \\
\hline Bilateral ovarian metastases & $0.864(0.527-1.233)$ & 0.320 & - & - \\
\hline Metastasectomy & $0.416(0.273-0.634)$ & $<0.001$ & $0.335(0.215-0.522)$ & $<0.001$ \\
\hline Peritoneal carcinomatosis & $2.584(1.695-3.937)$ & $<0.001$ & $2.212(1.387-3.534)$ & 0.001 \\
\hline Signet-ring cells & $2.597(1.658-4.065)$ & $<0.001$ & $1.938(1.182-3.175)$ & 0.009 \\
\hline Ascites & $1.565(1.035-2.364)$ & 0.034 & $1.062(0.686-1.672)$ & 0.798 \\
\hline Gastrectomy & $0.725(0.468-1.125)$ & 0.151 & - & - \\
\hline ER positive & $0.254(0.166-0.389)$ & $<0.001$ & $0.510(0.318-0.816)$ & 0.005 \\
\hline PR positive & $0.376(0.238-0.596)$ & $<0.001$ & $0.554(0.340-0.901)$ & 0.017 \\
\hline CA125 & $1.121(0.734-1.709)$ & 0.597 & - & - \\
\hline CEA & $1.292(0.810-2.062)$ & 0.282 & - & - \\
\hline CA199 & $1.541(1.026-2.316)$ & 0.037 & $1.208(0.774-1.886)$ & 0.406 \\
\hline
\end{tabular}


Additionally, 59.3\% (32/54) of the participants received R0 resection. Median OS in the R0 resection group was 22.6 (95\% CI 19.5-25.8) months as against 13.5 (95\% CI 11.0-16.0) months in the non-R0 resection group. Survival in the $\mathrm{R} 0$ resection group was significantly better than that in the non-R0 resection group $(P<0.001$; Figure 2).

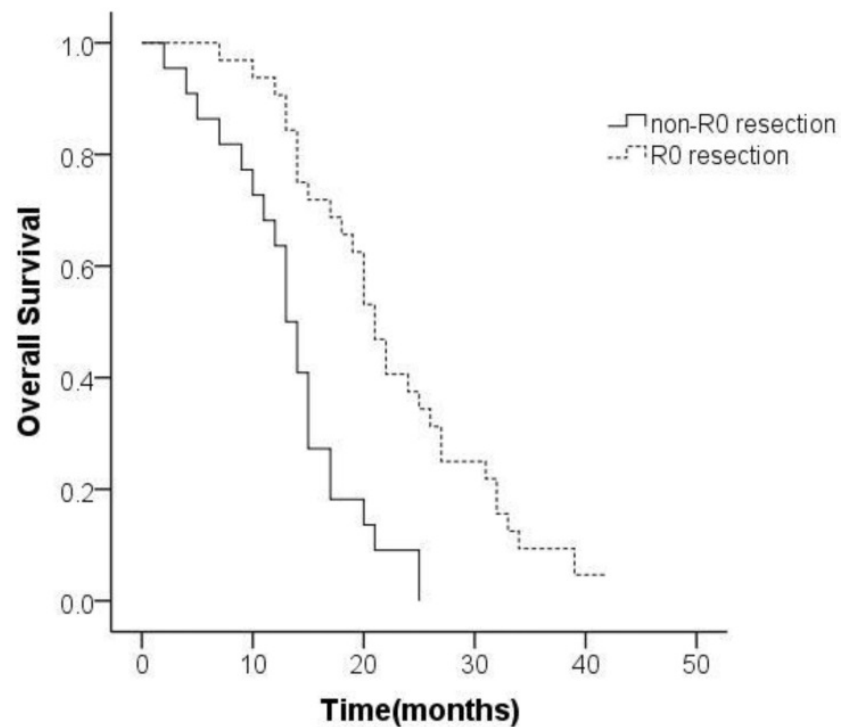

Figure 2. Kaplan-Meier analysis of overall survival based on curative resection of synchronous Krukenberg tumors from gastric cancer.

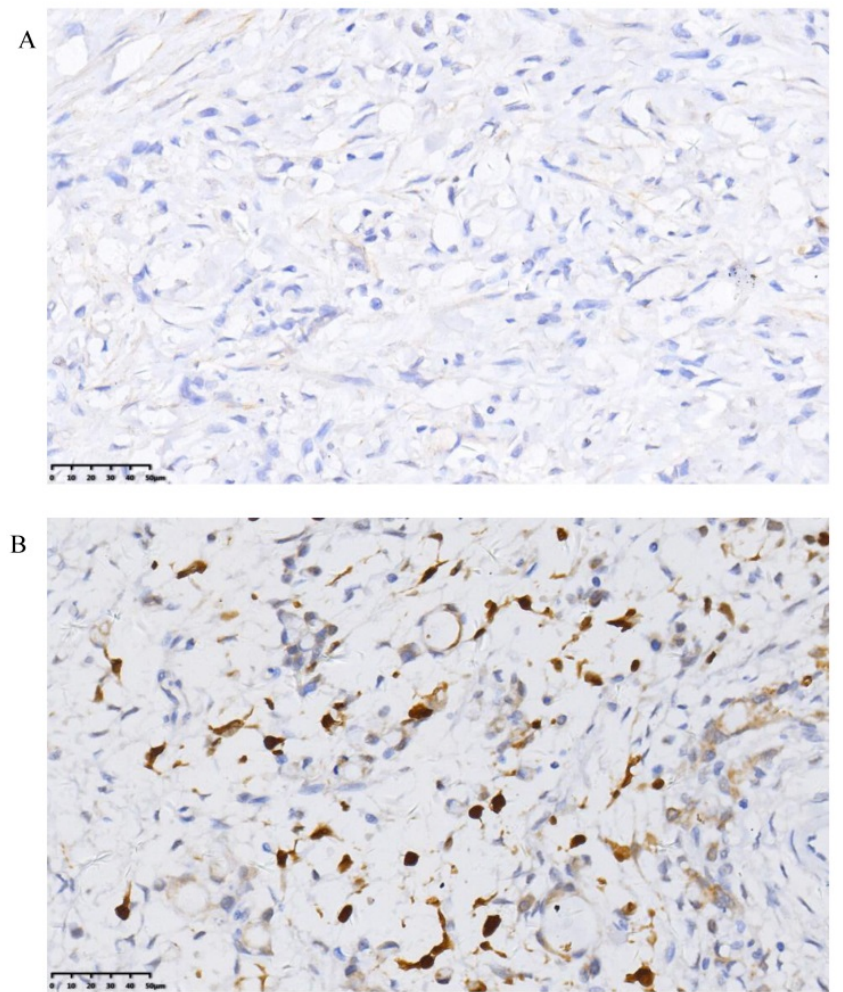

Figure 3. Representative images of negative (A) and positive (B) ER- $\beta$ expression.

\section{Association between expression of sex hormone receptors (SHR) and survival}

The positive rate of ER- $\beta$ was $44.7 \%$ and that of PR was $28.2 \%$. Representative histopathological images showing positive and negative expression of sex hormone receptors (SHR) were displayed in Figure 3 and Figure 4. Multivariate analysis revealed a positive association of OS with PR and ER- $\beta$ expression. The mean OS of ER- $\beta$-positive and -negative patients was 20.4 (95\% CI 18.1-22.7) months and 12.1 (95\% CI 10.4-13.9) months, respectively $(\mathrm{P}<0.001$; Figure 5). The mean OS of PR-positive and -negative patients was 20.6 (95\% CI 17.8-23.5) months and 13.8 (95\% CI 12.1-15.5) months, respectively $(\mathrm{P}=0.001$; Figure 6). These outcomes demonstrate a strong correlation between expressions of ER- $\beta$ and PR and survival of gastric cancer patients with synchronous ovarian metastases.
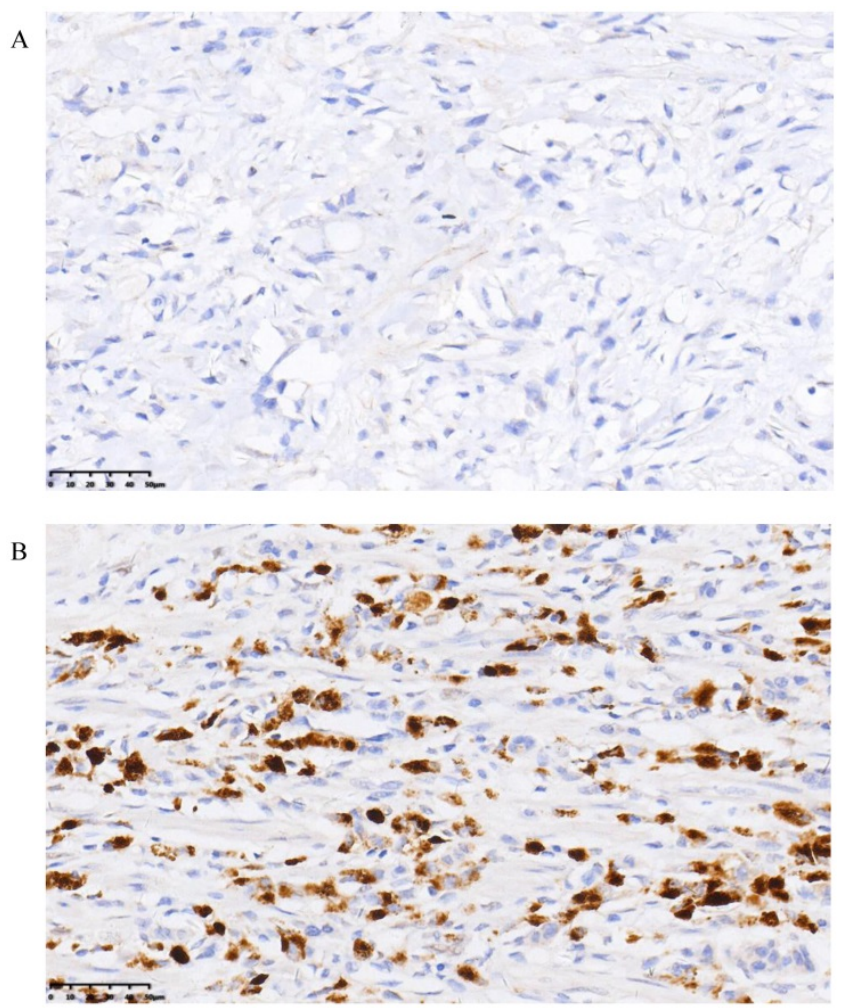

Figure 4. Representative images of negative (A) and positive (B) PR expression.

\section{Chemotherapy and adverse events}

Paclitaxel, S-1 and oxaliplatin were the most commonly used chemotherapeutic agents in this study population. Median duration of chemotherapy in the metastasectomy group (3.4 cycles, range 1-6 cycles) was shorter than that in the non-metastasectomy group (4.5 cycles, range 3-8 cycles); however, there was no statistical difference 
$(\mathrm{P}>0.05)$. Treatment-related adverse events and grade 3 or 4 toxicity occurred in $66.0 \%(68 / 103)$ and $35.9 \%$ $(37 / 103)$ of patients, respectively. Among hematological toxicity of grade 3 or 4 , leucopenia/neutropenia was the most frequent $(24.3 \%)$ followed by thrombocytopenia $(7.8 \%)$. The most common non-hematological toxicities were elevated levels of serum aspartate aminotransferase (3.9\%), elevated serum bilirubin concentrations (1.9\%) and rash $(1.9 \%)$.

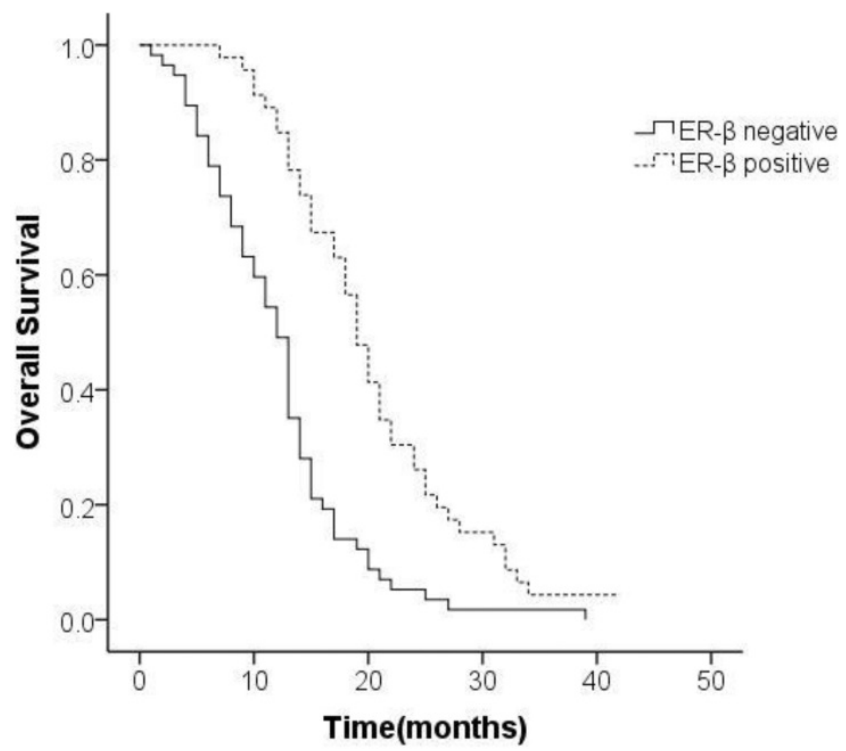

Figure 5. Survival curves of patients with expression status of ER- $\beta$.

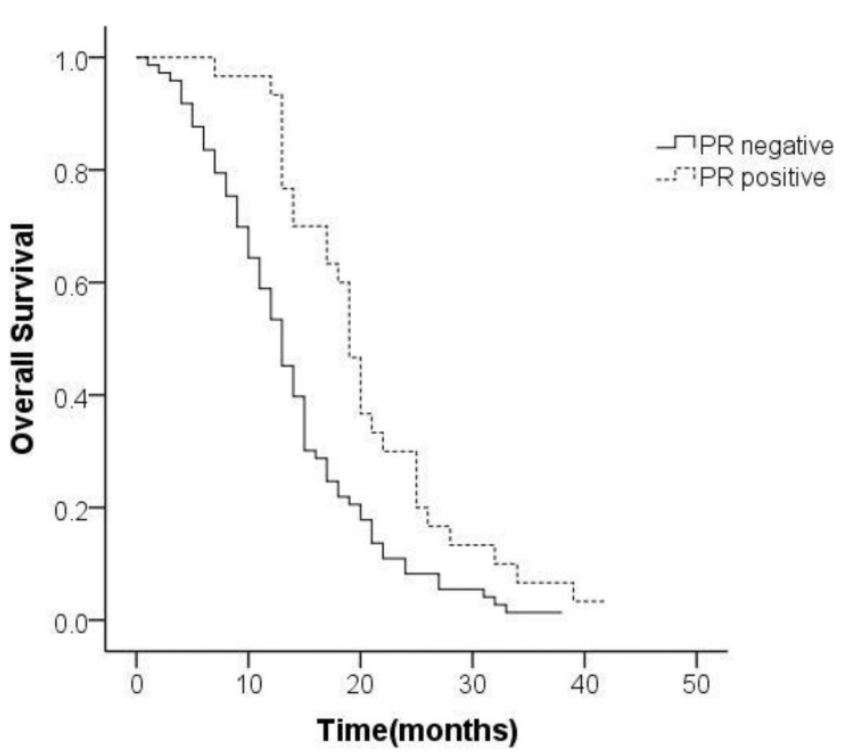

Figure 6. Survival curves of patients with expression status of PR.

\section{Postoperative complications}

A total of $9(16.7 \%)$ patients developed postoperative complications: anastomotic leakage $(n=3)$, pneumonia $(n=2)$, abdominal abscess $(n=2)$, delayed gastric emptying $(n=2)$, and hemorrhage $(n=1)$. In a vast majority of these patients, the complications were successfully resolved with conservative treatment; nevertheless, reoperation was performed in one patient due to intraperitoneal hemorrhage.

\section{Discussion}

The prognosis of ovarian metastasis originated from gastric carcinoma has been disclosed to be worse than that from other primary gastrointestinal tumors $[11,12]$. Although chemotherapy is one of the main therapeutic modalities for advanced gastric cancer with ovarian metastases, the curative effect has been unsatisfactory (reported median survival: 7-11 months) [13]. There have been very few reports with respect to surgical treatment. Some recent retrospective studies indicate that metastasectomy of Krukenberg tumors may positively influence the prognosis of these patients [7-9]. Nevertheless, a reasonable therapeutic strategy for Krukenberg tumors that originate from gastric cancer is yet to be established.

A retrospective study from $\mathrm{Lu}$ et al. analyzed 85 participants diagnosed as advanced gastric carcinoma with ovarian metastases since 2000 to 2010 .The results showed that the median survival in the resection group was 14.1 months as against 8 months in the non-resection group [7]. Another study in Korea reported superior OS of patients treated with metastasectomy plus chemotherapy as compared to that of patients who received chemotherapy alone (18.0 months vs. 8.0 months for patients with synchronous Krukenberg tumors). Nonetheless, the significance of the findings may be affected by the disproportion of the two groups [8].

In our series, outcomes of 103 gastric cancer patients with synchronous ovarian metastasis were retrospectively analyzed. Patients who underwent metastasetomy experienced significantly better OS as compared to their counterparts in the non-metastasetomy group. In both groups, the sample size was relatively large and the clinicopathological characteristics were comparable. Consistent with the results of previous studies, we conclude that metastasectomy should be the recommended strategy for gastric cancer patients with synchronous ovarian metastasis.

Evaluation of prognostic factors may help identify patients who could benefit from treatment. Independent risk factors for OS included metastasectomy, presence of signet ring cells, peritoneal carcinoma, and expressions of ER- $\beta$ and PR. Krukenberg tumors are often accompanied by variable degrees of peritoneal metastasis, which could result in ascites, intestinal obstruction, and cachexia, 
so as to adversely affect the quality of life of these patients [14]. Currently, there is no clear consensus on the optimal treatment strategy for gastric cancer with ovarian metastasis, particularly for patients with peritoneal metastases. Nonetheless, cytoreductive surgery plus chemotherapy may confer a survival benefit for these patients $[15,16]$. In our series, local peritoneal lesions were resected with ovariectomy and then followed by systemic chemotherapy. Further analysis displayed that the prognosis of patients with non-R0 resection was markedly worse than that of patients with R0 resection. Consequently, improved outcomes of Krukenberg tumors can be achieved with surgical resection without massive residual lesions. As one of the major therapeutics, chemotherapy could enhance living quality and prolong OS [17]. Combination or single agent chemotherapy (taxanes, platinum, fluoropyrimidine, and epirubicin) was shown to be effective for Krukenberg tumors with concomitant peritoneal dissemination $[18,19]$. A European study demonstrated the efficacy of hyperthermic intraperitoneal chemotherapy (HIPEC) in these patients [5]; we are also conducting a related clinical study (NCT02549911), and these need to be validated in the future.

Some investigations also suggest that the expression of SHR is associated with the occurrence as well as development of gastric cancer [20]. The protective role of sex hormones is important to female gastric cancer patients [21,22]. A recent meta-analysis showed that the risk of gastric cancer may decline during long-term exposure to estrogen [23]. However, the effect and significance of SHR in the context of Krukenberg tumors is not well characterized. After detection of the expressions of SHR in gastric cancer tissues, we noticed the presence of ER- $\beta$ and PR in tumor tissues with the absence of ER- $\alpha$. Our results indicated that positive expression of ER- $\beta$ or PR was related to better prognosis of gastric cancer patients with synchronous Krukenberg tumors. Available evidence suggests that SHR may be essential to the etiopathogenesis of Krukenberg tumors. Besides, it is not clear whether the incidence of ovarian metastasis from ER- or PR-positive gastric carcinoma can be reduced after targeted treatment for SHR. In conclusion, it is crucial to explore the implications of ER or PR expression for carcinogenesis and tumor progression in the setting of Krukenberg tumors of gastric origin in future.

The present study had several limitations. First, it was a retrospective, single center study. Further, the effect of some inherent selection bias on our analyses cannot be ruled out. Nevertheless, the relatively large sample size is a strength of our study. Moreover, this is the first study that evaluates the prognostic relevance of SHR in patients with synchronous Krukenberg tumors of gastric origin. Our findings may help facilitate the formulation of reasonable treatment strategies for these patients.

In our series, metastasectomy combined with appropriate chemotherapy was correlated with prolonged survival time for patients with synchronous Krukenberg tumors of gastric origin. Metastasectomy, presence of signet ring cells, peritoneal carcinomatosis, and expressions of ER- $\beta$ and PR were identified as prognostic factors for survival. Further studies are required to validate our conclusions, and establish reasonable treatment strategies for these patients.

\section{Abbreviations}

OS: overall survival; ER: estrogen receptor; PR: progesterone receptor; ECOG: Eastern Cooperative Oncology Group; PS: performance status; NUL: normal upper limit; CDSC: Clavien-Dindo severity classification; CTCAE: Common Terminology Criteria for Adverse Events; SHR: sex hormone receptors; HIPEC: hyperthermic intraperitoneal chemotherapy.

\section{Acknowledgements}

This study was supported by the Natural Science Foundation of Zhejiang Province of China (LY18H160032 to Pengfei Yu).

\section{Competing Interests}

The authors have declared that no competing interest exists.

\section{References}

1. Luo G, Zhang Y, Guo P, et al. Global patterns and trends in stomach cancer incidence: Age, period and birth cohort analysis. Int $\mathrm{J}$ Cancer. 2017;141(7):1333-44.

2. Matsushita $H$, Watanabe $K$, Wakatsuki A. Metastatic gastric cancer to the female genital tract. Mol Clin Oncol. 2016;5(5):495-99.

3. Wang J, Shi YK, Wu LY, et al. Prognostic factors for ovarian metastases from primary gastric cancer. Int J Gynecol Cancer. 2008;18(4):825-32.

4. Kim HK, Heo DS, Bang YJ, et al. Prognostic factors of Krukenberg's tumor. Gynecol Oncol .2001;82(1):105-9.

5. Rosa F, Marrelli D, Morgagni P, et al. Krukenberg Tumors of Gastric Origin: The Rationale of Surgical Resection and Perioperative Treatments in a Multicenter Western Experience. World J Surg. 2016;40(4):921-8.

6. Brieau B, Auzolle C, Pozet A, et al. Efficacy of modern chemotherapy and prognostic factors in patients with ovarian metastases from gastric cancer: A retrospective AGEO multicentre study. Dig Liver Dis. 2016;48(4):441-5.

7. Lu LC, Shao YY, Hsu CH, et al. Metastasectomy of Krukenberg tumors may be associated with survival benefits in patients with metastatic gastric cancer. Anticancer Res. 2012;32(8):3397-401.

8. Cho JH, Lim JY, Choi AR, et al. Comparison of Surgery Plus Chemotherapy and Palliative Chemotherapy Alone for Advanced Gastric Cancer with Krukenberg Tumor. Cancer Res Treat. 2015;47(4):697-705.

9. Peng W, Hua RX, Jiang $R$, et al. Surgical treatment for patients with Krukenberg tumor of stomach origin: clinical outcome and prognostic factors analysis. PLoS One. 2013;8(7):e68227.

10. Dindo D, Demartines N, Clavien PA. Classification of surgical complications: a new proposal with evaluation in a cohort of 6336 patients and results of a survey. Ann Surg. 2004;240(2):205-13.

11. Wu XJ, Yuan P, Li ZY, et al. Cytoreductive surgery and hyperthermic intraperitoneal chemotherapy improves the survival of gastric cancer patients with ovarian metastasis and peritoneal dissemination. Tumour Biol. 2013;34(1):463-9 
12. Ayhan A, Guvenal T, Salman MC, et al. The role of cytoreductive surgery in nongenital cancers metastatic to the ovaries. Gynecol Oncol. 2005;98(2):235-41.

13. Kiyokawa T, Young RH, Scully RE. Krukenberg tumors of the ovary: a clinicopathologic analysis of 120 cases with emphasis on their variable pathologic manifestations. Am J Surg Pathol. 2006;30(3):277-99.

14. Kobayashi O, Sugiyama Y, Cho H, et al. Clinical and pathological study of gastric cancer with ovarian metastasis. Int J Clin Oncol. 2003;8(2):67-71.

15. Feng $\mathrm{Q}$, Pei $\mathrm{W}$, Zheng $\mathrm{ZX}$, et al. Clinicopathologic characteristics and prognostic factors of 63 gastric cancer patients with metachronous ovarian metastasis. Cancer Biol Med. 2013;10(2):86-91.

16. Jun SY, Park JK. Metachronous ovarian metastases following resection of the primary gastric cancer. J Gastric Cancer. 2011;11(1):31-7.

17. Dahdaleh FS, Turaga KK. Evolving Treatment Strategies and Outcomes in Advanced Gastric Cancer with Peritoneal Metastasis. Surg Oncol Clin N Am. 2018;27(3):519-37.

18. Waddell T, Verheij M, Allum W, et al. Gastric cancer: ESMO-ESSO-ESTRO Clinical Practice Guidelines for diagnosis, treatment and follow-up. Ann Oncol. 2013;24 (Suppl 6):vi57-63.

19. Wagner AD, Unverzagt $S$, Grothe $W$, et al. Chemotherapy for advanced gastric cancer. Cochrane Database Syst Rev. 2010;(3):CD004064.

20. Wei S, Said-Al-Naief N, Hameed O. Estrogen and progesterone receptor expression is not always specific for mammary and gynecologic carcinomas: a tissue microarray and pooled literature review study. Appl Immunohistochem Mol Morphol. 2009;17(5):393-402.

21. Chandanos E, Lagergren J. Oestrogen and the enigmatic male predominance of gastric cancer. Eur J Cancer. 2008;44(16):2397-403.

22. Freedman ND, Ahn J, Hou L, et al. Polymorphisms in estrogen- and androgen-metabolizing genes and the risk of gastric cancer. Carcinogenesis. 2009;30(1):71-7.

23. Camargo MC, Goto $\mathrm{Y}$, Zabaleta J, et al. Sex hormones, hormonal interventions, and gastric cancer risk: a meta-analysis. Cancer Epidemiol Biomarkers Prev. 2012;21(1):20-38 